Article

\title{
An Extended VIKOR Method for Multiple Criteria Group Decision Making with Triangular Fuzzy Neutrosophic Numbers
}

\author{
Jie Wang, Guiwu Wei * ${ }^{-1}$ and Mao Lu * \\ School of Business, Sichuan Normal University, Chengdu 610101, China; JW970326@163.com \\ * Correspondence: weiguiwu1973@sicnu.edu.cn (G.W.); lumao@sicnu.edu.cn (M.L.)
}

Received: 1 August 2018; Accepted: 8 October 2018; Published: 15 October 2018

\begin{abstract}
In this article, we combine the original VIKOR model with a triangular fuzzy neutrosophic set to propose the triangular fuzzy neutrosophic VIKOR method. In the extended method, we use the triangular fuzzy neutrosophic numbers (TFNNs) to present the criteria values in multiple criteria group decision making (MCGDM) problems. Firstly, we summarily introduce the fundamental concepts, operation formulas and distance calculating method of TFNNs. Then we review some aggregation operators of TFNNs. Thereafter, we extend the original VIKOR model to the triangular fuzzy neutrosophic environment and introduce the calculating steps of the TFNNs VIKOR method, our proposed method which is more reasonable and scientific for considering the conflicting criteria. Furthermore, a numerical example for potential evaluation of emerging technology commercialization is presented to illustrate the new method, and some comparisons are also conducted to further illustrate advantages of the new method.
\end{abstract}

Keywords: MCGDM problems; triangular fuzzy neutrosophic sets (TFNSs); VIKOR model; TFNNs VIKOR method; potential evaluation; emerging technology commercialization

\section{Introduction}

The VIKOR (VIseKriterijumska Optimizacija I KOmpromisno Resenje) method [1] has been used to investigate multiple criteria group decision making (MCGDM) problems and has been widely used in many domains. In the existing literature, more and more traditional MCGDM models have been studied, such as: the grey relational analysis model [2-4]; the multi-objective optimization by ratio analysis plus the full multiplicative form (MULTIMOORA) model [5,6]; the Preference Ranking Organization Method for Enrichment of Evaluations (PROMETHEE) model [7]; the ELimination Et Choix Traduisant la REalité (ELECTRE) model [8]; and the Technique for Order of Preference by Similarity to Ideal Solution (TOPSIS) model [9,10].

In many real MCGDM problems, it is not easy to describe the criteria values with accurate values due to the fuzziness and complexity of the alternatives, and so it can be more effective and useful to describe the criteria values with fuzzy information. Fuzzy set theory [11] has been used as a feasible tool for MCGDM [12,13] problems. Smarandache [14,15] proposed the neutrosophic set (NS). Then, Wang et al. [16,17] defined the single-valued neutrosophic sets (SVNSs) and interval neutrosophic sets (INSs). Wang et al. [18,19] explored some aggregation operators of SVNNs and extended the SVNS to a 2-tuple linguistic neutrosophic number environment. Wu et al. [20] studied SVNNs with Hamy operators under 2-tuple linguistic neutrosophic numbers. Biswas et al. [21] provided the definition of a triangular fuzzy neutrosophic number (TFNN) in which the degree of truth-membership (MD), indeterminacy-membership (IMD) and falsity-membership (FMD) are depicted by TFNNs. Sahin et al. [22] studied multiple attribute decision making (MADM) problems 
with centroid single valued triangular neutrosophic numbers. Samah et al. [23] studied two ranking means based on information systems quality (ISQ) theory and the TFNNs environment. Ye [24] provided the definition of trapezoidal neutrosophic sets. Biswas et al. [25] studied some applications under the trapezoidal fuzzy neutrosophic environment. Tan and Zhang [26] defined some trapezoidal fuzzy neutrosophic aggregation operators.

Opricovic [1] used the VIKOR model to investigate some MCGDM problems with conflicting criteria [27,28]. Bausys and Zavadskas [29] established the INS VIKOR model. Liu and Park et al. [30] studied the VIKOR model under interval-valued intuitionistic fuzzy sets (IVIFSs). Selvakumari et al. [31] proposed the extended VIKOR model by constructing an octagonal neutrosophic soft matrix. Wan et al. [32] proposed the VIKOR model with triangular intuitionistic fuzzy numbers (TIFN), Liu et al. [33] provided the linguistic VIKOR model, and Qin et al. [34] developed the interval type-2 fuzzy VIKOR model. Chen [35] proposed the remoteness index-based Pythagorean fuzzy VIKOR methods with a generalized distance measure for multiple criteria decision analysis. Liao et al. [36] explored the VIKOR method with the hesitant fuzzy linguistic information. Ren et al. [37] provided the dual hesitant fuzzy VIKOR model. Li et al. [38] provided the VIKOR model with linguistic intuitionistic fuzzy numbers. Pouresmaeil et al. [39] established the SVNNs VIKOR model. Huang et al. [40] extended the VIKOR method to INSs. Zhang and Wei [41] extended the VIKOR method to a hesitant fuzzy environment.

However, there has been no study about the VIKOR model for MCGDM problems with TFNNs, so taking the TFNNs VIKOR model into account is of necessity. The goal of our article is to combine the original VIKOR model with TFNNs to study MCGDM problems. The structure of our paper is as follows. Section 1 introduces the concepts, operation formulas and the distance calculating method of TFNNs. Section 2 reviews some aggregation operators of TFNNs. Section 3 extends the original VIKOR model to a TFN environment and introduces the required calculating steps of TFNNs VIKOR method. Section 4 provides a numerical example for potential evaluation of emerging technology commercialization and introduces a comparison between our proposed methods and the existing method. Section 5 summarises our conclusions.

\section{Preliminaries}

\subsection{Triangular Fuzzy Neutrosophic Sets}

Based on the concepts of a traditional triangular fuzzy set and the fundamental theory of a single valued neutrosophic set (SVNS), the triangular fuzzy neutrosophic sets (TFNSs), which were first defined by Biswas et al., [21] can be depicted as follows:

Definition 1 [21]. Let $X$ be a fixed set. The TFNSs $\eta$ can be depicted as:

$$
\eta=\left\{\left(x, \phi_{\eta}(x), \varphi_{\eta}(x), \gamma_{\eta}(x)\right) \mid x \in X\right\}
$$

where $\phi_{\eta}(x), \varphi_{\eta}(x)$ and $\gamma_{\eta}(x) \in[0,1]$ represent the degree of the truth membership, the indeterminacy membership and the falsity membership, respectively, which can be expressed by triangular fuzzy numbers as follows.

$$
\begin{aligned}
& \phi_{\eta}(x)=\left(\phi_{\eta}^{L}(x), \phi_{\eta}^{M}(x), \phi_{\eta}^{U}(x)\right), 0 \leq \phi_{\eta}^{L}(x) \leq \phi_{\eta}^{M}(x) \leq \phi_{\eta}^{U}(x) \leq 1 \\
& \varphi_{\eta}(x)=\left(\varphi_{\eta}^{L}(x), \varphi_{\eta}^{M}(x), \varphi_{\eta}^{U}(x)\right), 0 \leq \varphi_{\eta}^{L}(x) \leq \varphi_{\eta}^{M}(x) \leq \varphi_{\eta}^{U}(x) \leq 1 \\
& \gamma_{\eta}(x)=\left(\gamma_{\eta}^{L}(x), \gamma_{\eta}^{M}(x), \gamma_{\eta}^{U}(x)\right), 0 \leq \gamma_{\eta}^{L}(x) \leq \gamma_{\eta}^{M}(x) \leq \gamma_{\eta}^{U}(x) \leq 1
\end{aligned}
$$


and the truth membership function can be defined:

$$
\phi_{\eta}(x)=\left\{\begin{array}{cc}
\frac{x-\phi_{\eta}^{L}(x)}{\phi_{\eta}^{M}(x)-\phi_{\eta}^{L}(x)}, & \phi_{\eta}^{L}(x) \leq x \leq \phi_{\eta}^{M}(x), \\
\frac{x-\phi_{\eta}^{U}(x)}{\phi_{\eta}^{M}(x)-\phi_{\eta}^{U}(x)}, & \phi_{\eta}^{M}(x) \leq x \leq \phi_{\eta}^{U}(x), \\
0, & \text { otherwise. }
\end{array}\right.
$$

For convenience, we let $\eta=\left\{\left(\phi^{L}, \phi^{M}, \phi^{U}\right),\left(\varphi^{L}, \varphi^{M}, \varphi^{U}\right),\left(\gamma^{L}, \gamma^{M}, \gamma^{U}\right)\right\}$ be a TFNN which satisfies the condition $0 \leq \phi^{U}+\varphi^{U}+\gamma^{U} \leq 3$.

Definition 2 [21]. Assume there are three TFNNs $\eta_{1}=\left\{\left(\phi_{1}^{L}, \phi_{1}^{M}, \phi_{1}^{U}\right),\left(\varphi_{1}^{L}, \varphi_{1}^{M}, \varphi_{1}^{U}\right),\left(\gamma_{1}^{L}, \gamma_{1}^{M}, \gamma_{1}^{U}\right)\right\}$, $\eta_{2}=\left\{\left(\phi_{2}^{L}, \phi_{2}^{M}, \phi_{2}^{U}\right),\left(\varphi_{2}^{L}, \varphi_{2}^{M}, \varphi_{2}^{U}\right),\left(\gamma_{2}^{L}, \gamma_{2}^{M}, \gamma_{2}^{U}\right)\right\}$ and $\eta=\left\{\left(\phi^{L}, \phi^{M}, \phi^{U}\right),\left(\varphi^{L}, \varphi^{M}, \varphi^{U}\right),\left(\gamma^{L}, \gamma^{M}, \gamma^{U}\right)\right\}$, the operation laws of them can be defined:

(1) $\eta_{1} \oplus \eta_{2}=\left\{\begin{array}{l}\left(\phi_{1}^{L}+\phi_{2}^{L}-\phi_{1}^{L} \phi_{2}^{L}, \phi_{1}^{M}+\phi_{2}^{M}-\phi_{1}^{M} \phi_{2}^{M}, \phi_{1}^{U}+\phi_{2}^{U}-\phi_{1}^{U} \phi_{2}^{U}\right),\left(\varphi_{1}^{L} \varphi_{2}^{L}, \varphi_{1}^{M} \varphi_{2}^{M}, \varphi_{1}^{U} \varphi_{2}^{U}\right), \\ \left(\gamma_{1}^{L} \gamma_{2}^{L}, \gamma_{1}^{M} \gamma_{2}^{M}, \gamma_{1}^{U} \gamma_{2}^{U}\right)\end{array}\right\} ;$

(2) $\eta_{1} \otimes \eta_{2}=\left\{\begin{array}{l}\left(\phi_{1}^{L} \phi_{2}^{L}, \phi_{1}^{M} \phi_{2}^{M}, \phi_{1}^{U} \phi_{2}^{U}\right),\left(\varphi_{1}^{L}+\varphi_{2}^{L}-\varphi_{1}^{L} \varphi_{2}^{L}, \varphi_{1}^{M}+\varphi_{2}^{M}-\varphi_{1}^{M} \varphi_{2}^{M}, \varphi_{1}^{U}+\varphi_{2}^{U}-\varphi_{1}^{U} \varphi_{2}^{U}\right), \\ \left(\gamma_{1}^{L}+\gamma_{2}^{L}-\gamma_{1}^{L} \gamma_{2}^{L}, \gamma_{1}^{M}+\gamma_{2}^{M}-\gamma_{1}^{M} \gamma_{2}^{M}, \gamma_{1}^{U}+\gamma_{2}^{U}-\gamma_{1}^{U} \gamma_{2}^{U}\right)\end{array}\right\} ;$

(3) $\lambda \eta=\left\{\begin{array}{l}\left(1-\left(1-\phi^{L}\right)^{\lambda}, 1-\left(1-\phi^{M}\right)^{\lambda}, 1-\left(1-\phi^{U}\right)^{\lambda}\right),\left(\left(\varphi^{L}\right)^{\lambda},\left(\varphi^{M}\right)^{\lambda},\left(\varphi^{U}\right)^{\lambda}\right), \\ \left(\left(\gamma^{L}\right)^{\lambda},\left(\gamma^{M}\right)^{\lambda},\left(\gamma^{U}\right)^{\lambda}\right)\end{array}\right\}, \lambda>0$;

(4) $\eta^{\lambda}=\left\{\begin{array}{l}\left(\left(\phi^{L}\right)^{\lambda},\left(\phi^{M}\right)^{\lambda},\left(\phi^{U}\right)^{\lambda}\right),\left(1-\left(1-\varphi^{L}\right)^{\lambda}, 1-\left(1-\varphi^{M}\right)^{\lambda}, 1-\left(1-\varphi^{U}\right)^{\lambda}\right), \\ \left(1-\left(1-\gamma^{L}\right)^{\lambda}, 1-\left(1-\gamma^{M}\right)^{\lambda}, 1-\left(1-\gamma^{U}\right)^{\lambda}\right)\end{array}\right\}, \lambda>0$.

According to Definition 2, it is clear that the operation laws have the following properties:

$$
\begin{gathered}
\eta_{1} \oplus \eta_{2}=\eta_{2} \oplus \eta_{1}, \quad \eta_{1} \otimes \eta_{2}=\eta_{2} \otimes \eta_{1}, \quad\left(\left(\eta_{1}\right)^{\lambda_{1}}\right)^{\lambda_{2}}=\left(\eta_{1}\right)^{\lambda_{1} \lambda_{2}} ; \\
\lambda\left(\eta_{1} \oplus \eta_{2}\right)=\lambda \eta_{1} \oplus \lambda \eta_{2}, \quad\left(\eta_{1} \otimes \eta_{2}\right)^{\lambda}=\left(\eta_{1}\right)^{\lambda} \otimes\left(\eta_{2}\right)^{\lambda} \\
\lambda_{1} \eta_{1} \oplus \lambda_{2} \eta_{1}=\left(\lambda_{1}+\lambda_{2}\right) \eta_{1}, \quad\left(\eta_{1}\right)^{\lambda_{1}} \otimes\left(\eta_{1}\right)^{\lambda_{2}}=\left(\eta_{1}\right)^{\left(\lambda_{1}+\lambda_{2}\right)} .
\end{gathered}
$$

Definition 3 [21]. Let $\eta=\left\{\left(\phi^{L}, \phi^{M}, \phi^{U}\right),\left(\varphi^{L}, \varphi^{M}, \varphi^{U}\right),\left(\gamma^{L}, \gamma^{M}, \gamma^{U}\right)\right\}$ be a TFNN, the score and accuracy functions of $\eta$ can be expressed:

$$
\begin{gathered}
s(\eta)=\frac{1}{12}\left[\begin{array}{l}
8+\left(\phi^{L}+2 \phi^{M}+\phi^{U}\right) \\
-\left(\gamma^{L}+2 \gamma^{M}+\gamma^{U}\right)
\end{array}\right],\left(\varphi^{L}+2 \varphi^{M}+\varphi^{U}\right) \\
h(\eta)=\frac{1}{4}\left[\left(\phi^{L}+2 \phi^{M}+\phi^{U}\right)-\left(\gamma^{L}+2 \gamma^{M}+\gamma^{U}\right)\right], h(\eta) \in[-1,1]
\end{gathered}
$$

Let $\eta_{1}$ and $\eta_{2}$ be two TFNNs. Then, based on Definition 3, the following assertion holds true.

(1) if $s\left(\eta_{1}\right)<s\left(\eta_{2}\right)$, then $\eta_{1}<\eta_{2}$;

(2) if $s\left(\eta_{1}\right)>s\left(\eta_{2}\right)$, then $\eta_{1}>\eta_{2}$;

(3) if $s\left(\eta_{1}\right)=s\left(\eta_{2}\right), h\left(\eta_{1}\right)<h\left(\eta_{2}\right)$, then $\eta_{1}<\eta_{2}$;

(4) if $s\left(\eta_{1}\right)=s\left(\eta_{2}\right), h\left(\eta_{1}\right)>h\left(\eta_{2}\right)$, then $\eta_{1}>\eta_{2}$;

(5) if $s\left(\eta_{1}\right)=s\left(\eta_{2}\right), h\left(\eta_{1}\right)=h\left(\eta_{2}\right)$, then $\eta_{1}=\eta_{2}$. 
2.2. The Normalized Hamming Distance between TFNNs

Definition 4 [32]. Let $\eta_{1}=\quad\left\{\left(\phi_{1}^{L}, \phi_{1}^{M}, \phi_{1}^{U}\right),\left(\varphi_{1}^{L}, \varphi_{1}^{M}, \varphi_{1}^{U}\right),\left(\gamma_{1}^{L}, \gamma_{1}^{M}, \gamma_{1}^{U}\right)\right\} \quad$ and $\eta_{2}=\left\{\left(\phi_{2}^{L}, \phi_{2}^{M}, \phi_{2}^{U}\right),\left(\varphi_{2}^{L}, \varphi_{2}^{M}, \varphi_{2}^{U}\right),\left(\gamma_{2}^{L}, \gamma_{2}^{M}, \gamma_{2}^{U}\right)\right\}$ be two TFNNs. Then the normalized Hamming distance is defined by:

$$
d\left(\eta_{1}, \eta_{2}\right)=\frac{1}{9}\left(\begin{array}{c}
\left|\phi_{1}^{L}-\phi_{2}^{L}\right|+\left|\phi_{1}^{M}-\phi_{2}^{M}\right|+\left|\phi_{1}^{U}-\phi_{2}^{U}\right| \\
+\left|\varphi_{1}^{L}-\varphi_{2}^{L}\right|+\left|\varphi_{1}^{M}-\varphi_{2}^{M}\right|+\left|\varphi_{1}^{U}-\varphi_{2}^{U}\right| \\
+\left|\gamma_{1}^{L}-\gamma_{2}^{L}\right|+\left|\gamma_{1}^{M}-\gamma_{2}^{M}\right|+\left|\gamma_{1}^{U}-\gamma_{2}^{U}\right|
\end{array}\right)
$$

Theorem 1. Assume that there are three TFNNs $\eta_{1}=\left\{\left(\phi_{1}^{L}, \phi_{1}^{M}, \phi_{1}^{U}\right),\left(\varphi_{1}^{L}, \varphi_{1}^{M}, \varphi_{1}^{U}\right),\left(\gamma_{1}^{L}, \gamma_{1}^{M}, \gamma_{1}^{U}\right)\right\}$, $\eta_{2}=\left\{\left(\phi_{2}^{L}, \phi_{2}^{M}, \phi_{2}^{U}\right),\left(\varphi_{2}^{L}, \varphi_{2}^{M}, \varphi_{2}^{U}\right),\left(\gamma_{2}^{L}, \gamma_{2}^{M}, \gamma_{2}^{U}\right)\right\}$ and $\eta=\left\{\left(\phi^{L}, \phi^{M}, \phi^{U}\right),\left(\varphi^{L}, \varphi^{M}, \varphi^{U}\right),\left(\gamma^{L}, \gamma^{M}, \gamma^{U}\right)\right\}$, the Hamming distance $d\left(\eta_{1}, \eta_{2}\right)$ has the following properties:

(P1) $0 \leq d\left(\eta_{1}, \eta_{2}\right) \leq 1$;

(P2) if $d\left(\eta_{1}, \eta_{2}\right)=0$, then $\eta_{1}=\eta_{2}$;

(P3) $d\left(\eta_{1}, \eta_{2}\right)=d\left(\eta_{2}, \eta_{1}\right)$;

(P4) $d\left(\eta_{1}, \eta_{2}\right)+d\left(\eta_{2}, \eta_{3}\right) \geq d\left(\eta_{1}, \eta_{3}\right)$.

Proof. (P1) $0 \leq d\left(\eta_{1}, \eta_{2}\right) \leq 1$

Since $0 \leq \phi^{L} \leq 1$, then $0 \leq\left|\phi_{1}^{L}-\phi_{2}^{L}\right| \leq 1$, similarly we see $0 \leq\left|\phi_{1}^{M}-\phi_{2}^{M}\right| \leq 1,0 \leq$ $\left|\phi_{1}^{U}-\phi_{2}^{U}\right| \leq 1,0 \leq\left|\varphi_{1}^{L}-\varphi_{2}^{L}\right| \leq 1,0 \leq\left|\varphi_{1}^{M}-\varphi_{2}^{M}\right| \leq 1,0 \leq\left|\varphi_{1}^{U}-\varphi_{2}^{U}\right| \leq 1,0 \leq\left|\gamma_{1}^{L}-\gamma_{2}^{L}\right| \leq$ $1,0 \leq\left|\gamma_{1}^{M}-\gamma_{2}^{M}\right| \leq 1,0 \leq\left|\gamma_{1}^{U}-\gamma_{2}^{U}\right| \leq 1$. So $0 \leq\left|\phi_{1}^{L}-\phi_{2}^{L}\right|+\left|\phi_{1}^{M}-\phi_{2}^{M}\right|+\left|\phi_{1}^{U}-\phi_{2}^{U}\right|+\left|\varphi_{1}^{L}-\varphi_{2}^{L}\right|+$ $\left|\varphi_{1}^{M}-\varphi_{2}^{M}\right|+\left|\varphi_{1}^{U}-\varphi_{2}^{U}\right|+\left|\gamma_{1}^{L}-\gamma_{2}^{L}\right|+\left|\gamma_{2}^{M}-\gamma_{1}^{M}\right|+\left|\gamma_{1}^{U}-\gamma_{2}^{U}\right| \leq 9$.

Therefore $0 \leq d\left(\eta_{1}, \eta_{2}\right) \leq 1$, which completes the proof.

(P2) if $d\left(\eta_{1}, \eta_{2}\right)=0$, then $\eta_{1}=\eta_{2}$

$$
\begin{aligned}
& d\left(\eta_{1}, \eta_{2}\right)=\frac{1}{9}\left(\begin{array}{c}
\left.\left|\phi_{1}^{L}-\phi_{2}^{L}\right|+\left|\phi_{1}^{M}-\phi_{2}^{M}\right|+\left|\phi_{1}^{U}-\phi_{2}^{U}\right|+\left|\varphi_{1}^{L}-\varphi_{2}^{L}\right|+\left|\varphi_{1}^{M}-\varphi_{2}^{M}\right|+\left|\varphi_{1}^{U}-\varphi_{2}^{U}\right|+\right)=0 \\
\left|\gamma_{1}^{L}\right|+\left|\gamma_{1}^{M}-\gamma_{2}^{M}\right|+\left|\gamma_{1}^{U}-\gamma_{2}^{U}\right|
\end{array}\right)=0,\left|\varphi_{1}^{L}-\varphi_{2}^{L}\right|=0,\left|\varphi_{1}^{M}-\varphi_{2}^{M}\right|=0,\left|\varphi_{2}^{U}\right|=0, \\
& \Rightarrow\left(\begin{array}{c}
\left|\phi_{1}^{L}-\phi_{2}^{L}\right|=0,\left|\phi_{1}^{M}-\phi_{2}^{M}\right|=0,\left|\phi_{1}^{U}-\phi_{2}^{U}\right|=0,\left|\varphi_{1}^{L}-\gamma_{2}^{L}-\gamma_{2}^{L}\right|=0,\left|\gamma_{1}^{M}-\gamma_{2}^{M}\right|=0,\left|\gamma_{1}^{U}-\gamma_{2}^{U}\right|=0 \\
\left|\gamma_{1}^{L}\right| \phi_{1}^{L}
\end{array}\right) \\
& \Rightarrow\left(\phi_{1}^{L}=\phi_{2}^{L}, \phi_{1}^{M}=\phi_{2}^{M}, \phi_{1}^{U}=\phi_{2}^{U}, \varphi_{1}^{L}=\varphi_{2}^{L}, \varphi_{1}^{M}=\varphi_{2}^{M}, \varphi_{1}^{U}=\varphi_{2}^{U}, \gamma_{1}^{L}=\gamma_{2}^{L}, \gamma_{1}^{M}=\gamma_{2}^{M}, \gamma_{1}^{U}=\gamma_{2}^{U}\right)
\end{aligned}
$$

That means $\eta_{1}=\eta_{2}$, and so (P2) if $d\left(\eta_{1}, \eta_{2}\right)=0$, then $\eta_{1}=\eta_{2}$ is correct.

(P3) $d\left(\eta_{1}, \eta_{2}\right)=d\left(\eta_{2}, \eta_{1}\right)$

$$
\begin{aligned}
& d\left(\eta_{1}, \eta_{2}\right)=\frac{1}{9}\left(\begin{array}{c}
\left|\phi_{1}^{L}-\phi_{2}^{L}\right|+\left|\phi_{1}^{M}-\phi_{2}^{M}\right|+\left|\phi_{1}^{U}-\phi_{2}^{U}\right|+\left|\varphi_{1}^{L}-\varphi_{2}^{L}\right|+\left|\varphi_{1}^{M}-\varphi_{2}^{M}\right|+\left|\varphi_{1}^{U}-\varphi_{2}^{U}\right| \\
+\left|\gamma_{1}^{L}-\gamma_{2}^{L}\right|+\left|\gamma_{1}^{M}-\gamma_{2}^{M}\right|+\left|\gamma_{1}^{U}-\gamma_{2}^{U}\right|
\end{array}\right) \\
& =\frac{1}{9}\left(\begin{array}{c}
\left|\phi_{2}^{L}-\phi_{1}^{L}\right|+\left|\phi_{2}^{M}-\phi_{1}^{M}\right|+\left|\phi_{2}^{U}-\phi_{1}^{U}\right|+\left|\varphi_{2}^{L}-\varphi_{1}^{L}\right|+\left|\varphi_{2}^{M}-\varphi_{1}^{M}\right|+\left|\varphi_{2}^{U}-\varphi_{1}^{U}\right| \\
+\left|\gamma_{2}^{L}-\gamma_{1}^{L}\right|+\left|\gamma_{2}^{M}-\gamma_{1}^{M}\right|+\left|\gamma_{2}^{U}-\gamma_{1}^{U}\right|
\end{array}\right)=d\left(\eta_{2}, \eta_{1}\right)
\end{aligned}
$$

So we complete the proof of (P3), which asserts that equality $d\left(\eta_{1}, \eta_{2}\right)=d\left(\eta_{2}, \eta_{1}\right)$ holds.

(P4) $d\left(\eta_{1}, \eta_{2}\right)+d\left(\eta_{2}, \eta_{3}\right) \geq d\left(\eta_{1}, \eta_{3}\right)$

$$
\begin{aligned}
& d\left(\eta_{1}, \eta_{3}\right)=\frac{1}{9}\left(\begin{array}{c}
\left|\phi_{1}^{L}-\phi_{3}^{L}\right|+\left|\phi_{1}^{M}-\phi_{3}^{M}\right|+\left|\phi_{1}^{U}-\phi_{3}^{U}\right|+\left|\varphi_{1}^{L}-\varphi_{3}^{L}\right|+\left|\varphi_{1}^{M}-\varphi_{3}^{M}\right| \\
+\left|\varphi_{1}^{U}-\varphi_{3}^{U}\right|+\left|\gamma_{1}^{L}-\gamma_{3}^{L}\right|+\left|\gamma_{1}^{M}-\gamma_{3}^{M}\right|+\left|\gamma_{1}^{U}-\gamma_{3}^{U}\right|
\end{array}\right) \\
& =\frac{1}{9}\left(\begin{array}{c}
\left|\phi_{1}^{L}-\phi_{2}^{L}+\phi_{2}^{L}-\phi_{3}^{L}\right|+\left|\phi_{1}^{M}-\phi_{2}^{M}+\phi_{2}^{M}-\phi_{3}^{M}\right|+\left|\phi_{1}^{U}-\phi_{2}^{U}+\phi_{2}^{U}-\phi_{3}^{U}\right| \\
+\left|\varphi_{1}^{L}-\varphi_{2}^{L}+\varphi_{2}^{L}-\varphi_{3}^{L}\right|+\left|\varphi_{1}^{M}-\varphi_{2}^{M}+\varphi_{2}^{M}-\varphi_{3}^{M}\right|+\left|\varphi_{1}^{U}-\varphi_{2}^{U}+\varphi_{2}^{U}-\varphi_{3}^{U}\right| \\
+\left|\gamma_{1}^{L}-\gamma_{2}^{L}+\gamma_{2}^{L}-\gamma_{3}^{L}\right|+\left|\gamma_{1}^{M}-\gamma_{2}^{M}+\gamma_{2}^{M}-\gamma_{3}^{M}\right|+\left|\gamma_{1}^{U}-\gamma_{2}^{U}+\gamma_{2}^{U}-\gamma_{3}^{U}\right|
\end{array}\right) \\
& \leq \frac{1}{9}\left(\begin{array}{l}
\left|\phi_{1}^{L}-\phi_{2}^{L}\right|+\left|\phi_{2}^{L}-\phi_{3}^{L}\right|+\left|\phi_{1}^{M}-\phi_{2}^{M}\right|+\left|\phi_{2}^{M}-\phi_{3}^{M}\right|+\left|\phi_{1}^{U}-\phi_{2}^{U}\right|+\left|\phi_{2}^{U}-\phi_{3}^{U}\right|+ \\
\left|\varphi_{1}^{L}-\varphi_{2}^{L}\right|+\left|\varphi_{2}^{L}-\varphi_{3}^{L}\right|+\left|\varphi_{1}^{M}-\varphi_{2}^{M}\right|+\left|\varphi_{2}^{M}-\varphi_{3}^{M}\right|+\left|\varphi_{1}^{U}-\varphi_{2}^{U}\right|+\left|\varphi_{2}^{U}-\varphi_{3}^{U}\right| \\
+\left|\gamma_{1}^{L}-\gamma_{2}^{L}\right|+\left|\gamma_{2}^{L}-\gamma_{3}^{L}\right|+\left|\gamma_{1}^{M}-\gamma_{2}^{M}\right|+\left|\gamma_{2}^{M}-\gamma_{3}^{M}\right|+\left|\gamma_{1}^{U}-\gamma_{2}^{U}\right|+\left|\gamma_{2}^{U}-\varphi_{3}^{U}\right|
\end{array}\right) \\
& =d\left(\eta_{1}, \eta_{2}\right)+d\left(\eta_{2}, \eta_{3}\right)
\end{aligned}
$$


Then, triangular fuzzy neutrosophic number weighted averaging (TFNNWA) and triangular fuzzy neutrosophic number weighted geometric (TFNNWG) operators are introduced as follows:

Definition 5 [21]. Let $\eta_{j}=\left\{\left(\phi_{j}^{L}, \phi_{j}^{M}, \phi_{j}^{U}\right),\left(\varphi_{j}^{L}, \varphi_{j}^{M}, \varphi_{j}^{U}\right),\left(\gamma_{j}^{L}, \gamma_{j}^{M}, \gamma_{j}^{U}\right)\right\}(j=1,2, \ldots, n)$ be a group of TFNNs, then the TFNNWA and TFNNWG operators proposed by Biswas et al. [21] are defined as follows.

$$
\operatorname{TFNNWA}\left(\eta_{1}, \eta_{2}, \ldots, \eta_{n}\right)=\omega_{1} \eta_{1} \oplus \omega_{2} \eta_{2} \ldots \oplus \omega_{n} \eta_{n}=\bigoplus_{j=1}^{n} \omega_{j} \eta_{j}
$$

and

$$
\operatorname{TFNNWG}\left(\eta_{1}, \eta_{2}, \ldots, \eta_{n}\right)=\left(\eta_{1}\right)^{\omega_{1}} \otimes\left(\eta_{2}\right)^{\omega_{2}} \ldots \otimes\left(\eta_{n}\right)^{\omega_{n}}=\bigotimes_{j=1}^{n}\left(\eta_{j}\right)^{\omega_{j}}
$$

where $\omega_{j}$ is weight vector of $\eta_{j}, j=1,2, \ldots, n$, which satisfies $0 \leq \omega_{j} \leq 1, \sum_{j=1}^{n} \omega_{j}=1$.

Theorem 2 [21]. Let $\eta_{j}=\left\{\left(\phi_{j}^{L}, \phi_{j}^{M}, \phi_{j}^{U}\right),\left(\varphi_{j}^{L}, \varphi_{j}^{M}, \varphi_{j}^{U}\right),\left(\gamma_{j}^{L}, \gamma_{j}^{M}, \gamma_{j}^{U}\right)\right\}(j=1,2, \ldots, n)$ be a group of TFNNs, then the operation results by TFNNWA and TFNNWG operators are also a TFNN where

$$
\begin{aligned}
& \operatorname{TFNNWA}\left(\eta_{1}, \eta_{2}, \ldots, \eta_{n}\right)=\stackrel{\oplus}{j=1}_{j}^{n} \omega_{j} \eta_{j} \\
& =\left\{\begin{array}{l}
\left(1-\prod_{j=1}^{n}\left(1-\phi_{j}^{L}\right)^{\omega_{j}}, 1-\prod_{j=1}^{n}\left(1-\phi_{j}^{M}\right)^{\omega_{j}}, 1-\prod_{j=1}^{n}\left(1-\phi_{j}^{U}\right)^{\omega_{j}}\right), \\
\left(\prod_{j=1}^{n}\left(\varphi_{j}^{L}\right)^{\omega_{j}}, \prod_{j=1}^{n}\left(\varphi_{j}^{M}\right)^{\omega_{j}}, \prod_{j=1}^{n}\left(\varphi_{j}^{U}\right)^{\omega_{j}}\right),\left(\prod_{j=1}^{n}\left(\gamma_{j}^{L}\right)^{\omega_{j}}, \prod_{j=1}^{n}\left(\gamma_{j}^{M}\right)^{\omega_{j}}, \prod_{j=1}^{n}\left(\gamma_{j}^{U}\right)^{\omega_{j}}\right)
\end{array}\right\}
\end{aligned}
$$

and

$$
\begin{aligned}
& \operatorname{TFNNWG}\left(\eta_{1}, \eta_{2}, \ldots, \eta_{n}\right)=\bigotimes_{j=1}^{n}\left(\eta_{j}\right)^{\omega_{j}} \\
& =\left\{\begin{array}{l}
\left(1-\prod_{j=1}^{n}\left(1-\varphi_{j}^{L}\right)^{\omega_{j}}, 1-\prod_{j=1}^{n}\left(1-\varphi_{j}^{M}\right)^{\omega_{j}}, 1-\prod_{j=1}^{n}\left(1-\varphi_{j}^{U}\right)^{\omega_{j}}\right), \\
\left(1-\prod_{j=1}^{n}\left(1-\gamma_{j}^{L}\right)^{\omega_{j}}, 1-\prod_{j=1}^{n}\left(1-\gamma_{j}^{M}\right)^{\omega_{j}}, 1-\prod_{j=1}^{n}\left(1-\gamma_{j}^{U}\right)^{\omega_{j}}\right),
\end{array}\right\}
\end{aligned}
$$

\subsection{VIKOR Method}

Denote $n$ alternatives under consideration as $O_{1}, O_{2}, \cdots, O_{n}$, the evaluation attribute as $C_{1}, C_{2}, \cdots, C_{n}$, and the rating of each alternative $O_{j}(j=1, \cdots, n)$ with respect to attribute $C_{j}(j=1, \cdots, m)$ as $f_{i j}$. Then the compromise ranking algorithm of the VIKOR method [42-45] has the following steps:

Step 1. Determine the best rating $f_{i}^{+}$and the worst rating $f_{i}^{-}$for all the attributes. For example, it the attribute $i$ represents a benefit, then

$$
f_{i}^{+}=\min _{j} f_{i j}, f_{i}^{-}=\min _{j} f_{i j}
$$

Naturally, a candidate having scores $\left(f_{1}^{+}, f_{2}^{+}, \ldots, f_{m}^{+}\right)$would be positive ideal whereas a candidate having scores $\left(f_{1}^{-}, f_{2}^{-}, \cdots, f_{m}^{-}\right)$would be a negative ideal candidate. It is assumed that such a positive ideal candidate does not exist; otherwise, the decision would be trivial. 
Step 2. Compute the values and $S_{j}$ and $R_{j}(j=1, \cdots, n)$ which represent the average and the worst group scores of the alternatives $O_{j}$, respectively, with the relations

$$
\begin{gathered}
S_{j}=\sum_{i=1}^{n} w_{i} \frac{\left(f_{i}^{+}-f_{i j}\right)}{\left(f_{i}^{+}-f_{i}^{-}\right)}, S_{j} \in[0,1] \\
R_{j}=\max _{i}\left[w_{i} \frac{\left(f_{i}^{+}-f_{i j}\right)}{\left(f_{i}^{+}-f_{i}^{-}\right)}\right], R_{j}=[0,1]
\end{gathered}
$$

Here, $w_{j}\left(\sum_{i=1}^{m} w_{i}=1, w_{i} \in[0,1], i=1,2, \cdots, m\right)$ is the relative importance weights of the criteria set by the decision maker. The smaller values of $S_{j}$ and $R_{j}$ correspond to the better, average and worse group scores of alternatives $O_{j}$, respectively.

Step 3. Compute the $Q_{j}$ values for $j=1,2, \cdots, m$ with the relation

$$
Q_{j}=\frac{\alpha\left(S_{j}-S^{+}\right)}{\left(S^{-}-S^{+}\right)}+\frac{(1-\alpha)\left(R_{j}-R^{+}\right)}{\left(R^{-}-R^{+}\right)}
$$

where

$$
\begin{aligned}
S^{+} & =\min _{j} S_{j}, S^{-}=\max _{j} S_{j} \\
R^{+} & =\min _{j} R_{j}, R^{-}=\max _{j} R_{j}
\end{aligned}
$$

and $\alpha$ is the weight of decision making strategy "the majority of attribute" (or "the maximum group utility"). The compromise can be selected with "voting by majority" $(\alpha>0.5)$, with "consensus" $(\alpha=0.5)$, with "veto" $(\alpha<0.5)$.

Step 4. Rank the alternatives by sorting each $S, R$ and $Q$ values in a decreasing order. The result is a set of three ranking lists denoted as $S_{[.]}, R_{[.]}$and $Q_{[.]}$.

Step 5. Propose the alternative $O_{j 1}$ corresponding to $O_{[1]}$ (the smallest among $Q_{j}$ values) as compromise solution if

C1. The alternative $O_{j 1}$ has an acceptable advantage; in other words, $Q_{[2]}-Q_{[1]} \geq D Q$ where $D Q=\frac{1}{(m-1)}$, and $m$ is the number of alternatives.

C2. The alternative $O_{j 1}$ is stable within the decision making process; in other words, it is also the best ranked in $S_{[.]}$or $R_{[\cdot]}$.

If one of the above conditions is not satisfied, then a set of compromise solutions is proposed, which consists of:

- Alternatives $O_{j 1}$ and $O_{j 2}$ where $Q_{j 2}=Q_{[2]}$ if only the condition is not satisfied, or

- Alternatives $O_{j 1}, O_{j 2}, \cdots, O_{j k}$ if the condition $C_{1}$ is not satisfied; and $O_{j k}$ is determined by the relation $Q_{k}-Q_{[1]}<D Q$ for the maximum $k$ where $Q_{j k}=Q_{[k]}$ (the positions of these alternatives are in closeness).

\section{VIKOR Model for MCGDM Problems with TFNNs}

Let $\left\{\varphi_{1}, \varphi_{2}, \ldots \varphi_{m}\right\}$ be a group of alternatives, $\left\{d_{1}, d_{2}, \ldots d_{t}\right\}$ be a list of experts with weighting vector being $\left\{v_{1}, v_{2}, \ldots v_{t}\right\}$, and $\left\{c_{1}, c_{2}, \ldots c_{n}\right\}$ be a list of criteria with weighting vector being $\left\{\omega_{1}, \omega_{2}, \ldots \omega_{n}\right\}$, which thereby satisfies $\omega_{i} \in[0,1], v_{\lambda} \in[0,1]$ and $\sum_{i=1}^{n} \omega_{i}=1, \sum_{\lambda=1}^{t} v_{\lambda}=1$. Construct the evaluation matrixes $\eta^{\lambda}=\left[\eta_{i j}^{\lambda}\right]_{m \times n}$ where $\eta_{i j}^{\lambda}=\left\{\left(\left(\phi_{i j}^{L}\right)^{\lambda},\left(\phi_{i j}^{M}\right)^{\lambda},\left(\phi_{i j}^{U}\right)^{\lambda}\right),\left(\left(\varphi_{i j}^{L}\right)^{\lambda},\left(\varphi_{i j}^{M}\right)^{\lambda},\left(\varphi_{i j}^{U}\right)^{\lambda}\right),\left(\left(\gamma_{i j}^{L}\right)^{\lambda},\left(\gamma_{i j}^{M}\right)^{\lambda},\left(\gamma_{i j}^{U}\right)^{\lambda}\right)\right\}$ means the estimate results of the alternative $\varphi_{i}(i=1,2, \ldots, m)$ based on the criterion $c_{j}(j=1,2, \ldots, n)$ by expert $d_{\lambda}$. Let $\left(\left(\phi_{i j}^{L}\right)^{\lambda},\left(\phi_{i j}^{M}\right)^{\lambda},\left(\phi_{i j}^{U}\right)^{\lambda}\right) \in[0,1]$ denote the degree of truth-membership (TMD), 
$\left(\left(\varphi_{i j}^{L}\right)^{\lambda},\left(\varphi_{i j}^{M}\right)^{\lambda},\left(\varphi_{i j}^{U}\right)^{\lambda}\right) \in[0,1]$ denote the degree of indeterminacy-membership (IMD) and $\left(\left(\gamma_{i j}^{L}\right)^{\lambda},\left(\gamma_{i j}^{M}\right)^{\lambda},\left(\gamma_{i j}^{U}\right)^{\lambda}\right) \in[0,1]$ denote the degree of falsity-membership (FMD) $0 \leq\left(\phi_{i j}^{U}\right)^{\lambda}+$ $\left(\varphi_{i j}^{U}\right)^{\lambda}+\left(\gamma_{i j}^{u}\right)^{\lambda} \leq 3 i=1,2, \ldots, m, j=1,2, \ldots, n, \lambda=1,2, \ldots, t$.

Considering both the TFNNs theories and the traditional VIKOR model, we try to propose a TFNNs VIKOR model to study MCGDM problems effectively. The model can be depicted as follows:

Step 1. Construct the decision matrixes $\eta^{\lambda}=\left[\eta_{i j}^{\lambda}\right]_{m \times n^{\prime}}$, and utilize overall values of $\eta^{\lambda}=\left[\eta_{i j}^{\lambda}\right]_{m \times n}$ to $\eta=\left[\eta_{i j}\right]_{m \times n}$ by using equal (14) or (15);

Step 2. Compute the positive ideal solution (PIS) $\varphi^{+}$and the negative ideal solution (NIS) $\varphi^{-}$;

$$
\begin{gathered}
\varphi^{+}=\left\{\left(\left(\phi_{j}^{L}\right)^{+},\left(\phi_{j}^{M}\right)^{+},\left(\phi_{j}^{U}\right)^{+}\right),\left(\left(\varphi_{j}^{L}\right)^{+},\left(\varphi_{j}^{M}\right)^{+},\left(\varphi_{j}^{U}\right)^{+}\right),\left(\left(\gamma_{j}^{L}\right)^{+},\left(\gamma_{j}^{M}\right)^{+},\left(\gamma_{j}^{U}\right)^{+}\right)\right\} \\
\varphi^{-}=\left\{\left(\left(\phi_{j}^{L}\right)^{-},\left(\phi_{j}^{M}\right)^{-},\left(\phi_{j}^{U}\right)^{-}\right),\left(\left(\varphi_{j}^{L}\right)^{-},\left(\varphi_{j}^{M}\right)^{-},\left(\varphi_{j}^{U}\right)^{-}\right),\left(\left(\gamma_{j}^{L}\right)^{-},\left(\gamma_{j}^{M}\right)^{-},\left(\gamma_{j}^{U}\right)^{-}\right)\right\}
\end{gathered}
$$

For benefit attribute

$$
\begin{aligned}
& \left\{\begin{array}{l}
\left(\left(\phi_{j}^{L}\right)^{+},\left(\phi_{j}^{M}\right)^{+},\left(\phi_{j}^{U}\right)^{+}\right), \\
\left(\left(\varphi_{j}^{L}\right)^{+},\left(\varphi_{j}^{M}\right)^{+},\left(\varphi_{j}^{U}\right)^{+}\right), \\
\left(\left(\gamma_{j}^{L}\right)^{+},\left(\gamma_{j}^{M}\right)^{+},\left(\gamma_{j}^{U}\right)^{+}\right)
\end{array}\right\}=\left\{\begin{array}{l}
\left(\max _{i}\left(\phi_{i j}^{L}\right), \max _{i}\left(\phi_{i j}^{M}\right), \max _{i}\left(\phi_{i j}^{U}\right)\right), \\
\left(\min _{i}\left(\varphi_{i j}^{L}\right), \min _{i}\left(\varphi_{i j}^{M}\right), \min _{i}\left(\varphi_{i j}^{U}\right)\right), \\
\left(\min _{i}\left(\gamma_{i j}^{L}\right), \min _{i}\left(\gamma_{i j}^{M}\right), \min _{i}\left(\gamma_{i j}^{U}\right)\right)
\end{array}\right\} \\
& \left\{\begin{array}{l}
\left(\left(\phi_{j}^{L}\right)^{-},\left(\phi_{j}^{M}\right)^{-},\left(\phi_{j}^{U}\right)^{-}\right), \\
\left(\left(\varphi_{j}^{L}\right)^{-},\left(\varphi_{j}^{M}\right)^{-},\left(\varphi_{j}^{U}\right)^{-}\right), \\
\left(\left(\gamma_{j}^{L}\right)^{-},\left(\gamma_{j}^{M}\right)^{-},\left(\gamma_{j}^{U}\right)^{-}\right)
\end{array}\right\}=\left\{\begin{array}{l}
\left(\min _{i}\left(\phi_{i j}^{L}\right), \min _{i}\left(\phi_{i j}^{M}\right), \min _{i}\left(\phi_{i j}^{U}\right)\right), \\
\left(\max _{i}\left(\varphi_{i j}^{L}\right), \max _{i}\left(\varphi_{i j}^{M}\right), \max _{i}\left(\varphi_{i j}^{U}\right)\right), \\
\left(\max _{i}\left(\gamma_{i j}^{L}\right), \max _{i}\left(\gamma_{i j}^{M}\right), \max _{i}\left(\gamma_{i j}^{U}\right)\right)
\end{array}\right\}
\end{aligned}
$$

For cost attribute

$$
\begin{gathered}
\left\{\begin{array}{l}
\left(\left(\phi_{j}^{L}\right)^{+},\left(\phi_{j}^{M}\right)^{+},\left(\phi_{j}^{U}\right)^{+}\right), \\
\left(\left(\varphi_{j}^{L}\right)^{+},\left(\varphi_{j}^{M}\right)^{+},\left(\varphi_{j}^{U}\right)^{+}\right), \\
\left(\left(\gamma_{j}^{L}\right)^{+},\left(\gamma_{j}^{M}\right)^{+},\left(\gamma_{j}^{U}\right)^{+}\right)
\end{array}\right\}=\left\{\begin{array}{l}
\left(\min _{i}\left(\phi_{i j}^{L}\right), \min _{i}\left(\phi_{i j}^{M}\right), \min _{i}\left(\phi_{i j}^{U}\right)\right), \\
\left(\max _{i}\left(\varphi_{i j}^{L}\right), \max _{i}\left(\varphi_{i j}^{M}\right), \max _{i}\left(\varphi_{i j}^{U}\right)\right), \\
\left(\max _{i}\left(\gamma_{i j}^{L}\right), \max _{i}\left(\gamma_{i j}^{M}\right), \max _{i}\left(\gamma_{i j}^{U}\right)\right)
\end{array}\right\} \\
\left\{\begin{array}{l}
\left(\left(\phi_{j}^{L}\right)^{-},\left(\phi_{j}^{M}\right)^{-},\left(\phi_{j}^{U}\right)^{-}\right), \\
\left(\left(\varphi_{j}^{L}\right)^{-},\left(\varphi_{j}^{M}\right)^{-},\left(\varphi_{j}^{U}\right)^{-}\right), \\
\left(\left(\gamma_{j}^{L}\right)^{-},\left(\gamma_{j}^{M}\right)^{-},\left(\gamma_{j}^{U}\right)^{-}\right)
\end{array}\right\}=\left\{\begin{array}{l}
\left(\max _{i}\left(\phi_{i j}^{L}\right), \max _{i}\left(\phi_{i j}^{M}\right), \max _{i}\left(\phi_{i j}^{U}\right)\right), \\
\left(\min _{i}\left(\varphi_{i j}^{L}\right), \min _{i}\left(\varphi_{i j}^{M}\right), \min _{i}\left(\varphi_{i j}^{U}\right)\right), \\
\left(\min _{i}\left(\gamma_{i j}^{L}\right), \min _{i}\left(\gamma_{i j}^{M}\right), \min _{i}\left(\gamma_{i j}^{U}\right)\right)
\end{array}\right\}
\end{gathered}
$$


Step 3. Based on Equation (11) and the attribute weighting vector $\omega_{j}$, we can calculate the values of $\chi_{i}$ and $\psi_{i}$ which express the average and the worst group scores of $\varphi_{i}$.

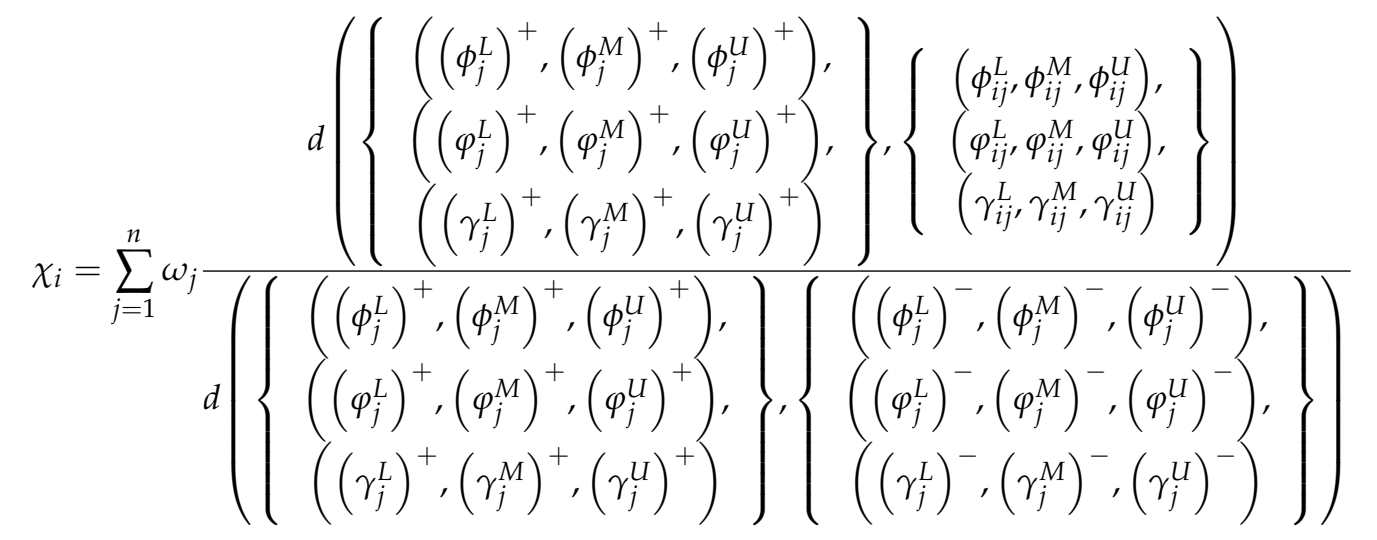

$$
\begin{aligned}
& \psi_{i}=\max _{j}\left\{\begin{array}{r}
\left.\left\{\begin{array}{l}
\left(\left(\phi_{j}^{L}\right)^{+},\left(\phi_{j}^{M}\right)^{+},\left(\phi_{j}^{U}\right)^{+}\right), \\
\left(\left(\varphi_{j}^{L}\right)^{+},\left(\varphi_{j}^{M}\right)^{+},\left(\varphi_{j}^{U}\right)^{+}\right), \\
\left(\left(\gamma_{j}^{L}\right)^{+},\left(\gamma_{j}^{M}\right)^{+},\left(\gamma_{j}^{U}\right)^{+}\right.
\end{array}\right\},\left\{\begin{array}{r}
\left(\phi_{i j}^{L}, \phi_{i j}^{M}, \phi_{i j}^{U}\right), \\
\left(\varphi_{i j}^{L}, \varphi_{i j}^{M}, \varphi_{i j}^{U}\right), \\
\left(\gamma_{i j}^{L}, \gamma_{i j}^{M}, \gamma_{i j}^{U}\right)
\end{array}\right\}\right) \\
d\left(\left\{\begin{array}{l}
\left(\left(\phi_{j}^{L}\right)^{+},\left(\phi_{j}^{M}\right)^{+},\left(\phi_{j}^{U}\right)^{+}\right), \\
\left(\left(\varphi_{j}^{L}\right)^{+},\left(\varphi_{j}^{M}\right)^{+},\left(\varphi_{j}^{U}\right)^{+}\right), \\
\left(\left(\gamma_{j}^{L}\right)^{+},\left(\gamma_{j}^{M}\right)^{+},\left(\gamma_{j}^{U}\right)^{+}\right)
\end{array}\right\}\left\{\begin{array}{l}
\left(\left(\phi_{j}^{L}\right)^{-},\left(\phi_{j}^{M}\right)^{-},\left(\phi_{j}^{U}\right)^{-}\right), \\
\left(\left(\varphi_{j}^{L}\right)^{-},\left(\varphi_{j}^{M}\right)^{-},\left(\varphi_{j}^{U}\right)^{-}\right), \\
\left(\left(\gamma_{j}^{L}\right)^{-},\left(\gamma_{j}^{M}\right)^{-},\left(\gamma_{j}^{U}\right)^{-}\right)
\end{array}\right\}\right)
\end{array}\right\}
\end{aligned}
$$

where $d$ is the normalized Hamming distance and $0 \leq \omega_{j} \leq 1$ means the weight of attributes which satisfies $\sum_{i=1}^{n} \omega_{i}=1$.

Step 4. Compute the values of $\Omega_{i}$ based on the results of $\chi_{i}$ and $\psi_{i}$, the calculating formula is characterized as follows:

$$
\Omega_{i}=\alpha \frac{\left(\chi_{i}-\chi^{+}\right)}{\left(\chi^{-}-\chi^{+}\right)}+(1-\alpha) \frac{\left(\psi_{i}-\psi^{+}\right)}{\left(\psi^{-}-\psi^{+}\right)}
$$

where

$$
\begin{aligned}
& \chi^{+}=\min _{i} \chi_{i}, \chi^{-}=\max _{i} \chi_{i} \\
& \psi^{+}=\min _{i} \psi_{i}, \psi^{-}=\max _{i} \psi_{i}
\end{aligned}
$$

where $\alpha$ means the coefficient of decision making strategic. $\alpha>0.5$ depicts "the maximum group utility", $\alpha=0.5$ depicts equality and $\alpha<0.5$ depicts the minimum regret.

Step 5. To choose the best alternative in accordance with the values of $\Omega_{i}$, the alternative with minimum value is the best choice.

\section{Numerical Example}

\subsection{Calculating Steps Based on MCGDM Problems}

In this section we present a numerical example to show potential evaluation of emerging technology commercialization with TFNNs in order to illustrate the method proposed in this paper. There is a panel with five possible emerging technology enterprises $\varphi_{i}(i=1,2,3,4,5)$ to select from. The experts select four criteria to evaluate the five possible emerging technology enterprises: (1) $c_{1}$ stands for the technical advancement; (2) $c_{2}$ stands for the potential market and market risk; (3) $c_{3}$ stands for the industrialization infrastructure, human resources and financial conditions; (4) $c_{4}$ stands 
for the employment creation and the development of science and technology. The five possible emerging technology enterprises $\varphi_{i}(i=1,2,3,4,5)$ are to be evaluated using the TFNNs with the four criteria by three experts $d_{\lambda}(\lambda=1,2,3)$ (criteria weight $\omega=(0.42,0.13,0.25,0.30)$, experts weight $v=(0.35,0.45,0.20)$.$) , which are given in Tables 1-3$.

Table 1. Triangular fuzzy neutrosophic numbers (TFNNs) evaluation matrix by $d_{1}$.

\begin{tabular}{|c|c|c|c|c|}
\hline & $c_{1}$ & $c_{2}$ & $c_{3}$ & $\mathrm{c}_{4}$ \\
\hline$\varphi_{1}$ & $\begin{array}{c}(0.6,0.8,0.9) \\
(0.2,0.3,0.5) \\
(0.1,0.3,0.4)\end{array}$ & $\begin{array}{c}(0.3,0.5,0.7) \\
(0.4,0.5,0.6) \\
(0.2,0.4,0.5)\end{array}$ & $\begin{array}{c}(0.5,0.6,0.7) \\
(0.1,0.2,0.3) \\
(0.2,0.3,0.4)\end{array}$ & $\begin{array}{c}(0.4,0.7,0.9), \\
(0.5,0.6,0.8), \\
(0.2,0.4,0.6)\end{array}$ \\
\hline$\varphi_{2}$ & $\begin{array}{c}(0.5,0.6,0.7) \\
(0.4,0.5,0.6) \\
(0.3,0.4,0.5)\end{array}$ & $\begin{array}{c}(0.2,0.4,0.6) \\
(0.3,0.5,0.7) \\
(0.2,0.3,0.5)\end{array}$ & $\begin{array}{c}(0.5,0.6,0.9) \\
(0.6,0.7,0.8) \\
(0.5,0.6,0.7)\end{array}$ & $\begin{array}{c}(0.4,0.5,0.7), \\
(0.3,0.4,0.6), \\
(0.5,0.6,0.8)\end{array}$ \\
\hline$\varphi_{3}$ & $\begin{array}{c}(0.3,0.5,0.6) \\
(0.2,0.4,0.5) \\
(0.5,0.6,0.8)\end{array}$ & $\begin{array}{c}(0.2,0.3,0.4) \\
(0.4,0.5,0.6) \\
(0.6,0.7,0.8)\end{array}$ & $\begin{array}{c}(0.7,0.8,0.9) \\
(0.3,0.5,0.6) \\
(0.2,0.4,0.5)\end{array}$ & $\begin{array}{c}(0.4,0.5,0.8), \\
(0.5,0.7,0.9), \\
(0.2,0.3,0.4)\end{array}$ \\
\hline$\varphi_{4}$ & $\begin{array}{c}(0.2,0.5,0.7) \\
(0.3,0.6,0.8) \\
(0.1,0.2,0.3)\end{array}$ & $\begin{array}{c}(0.5,0.7,0.8) \\
(0.3,0.4,0.5) \\
(0.2,0.5,0.7)\end{array}$ & $\begin{array}{c}(0.4,0.6,0.8) \\
(0.3,0.4,0.5) \\
(0.2,0.3,0.6)\end{array}$ & $\begin{array}{c}(0.5,0.7,0.9), \\
(0.4,0.6,0.8), \\
(0.2,0.3,0.5)\end{array}$ \\
\hline$\varphi_{5}$ & $\begin{array}{c}(0.7,0.8,0.9) \\
(0.2,0.3,0.4) \\
(0.2,0.3,0.4)\end{array}$ & $\begin{array}{c}(0.5,0.7,0.8) \\
(0.4,0.5,0.8) \\
(0.2,0.4,0.5)\end{array}$ & $\begin{array}{c}(0.5,0.6,0.7) \\
(0.2,0.4,0.5) \\
(0.1,0.4,0.6)\end{array}$ & $\begin{array}{c}(0.3,0.4,0.9) \\
(0.2,0.4,0.5), \\
(0.1,0.5,0.6)\end{array}$ \\
\hline
\end{tabular}

Table 2. TFNNs evaluation matrix by $d_{2}$.

\begin{tabular}{|c|c|c|c|c|}
\hline & $c_{1}$ & $\mathrm{c}_{2}$ & $c_{3}$ & $\mathrm{c}_{4}$ \\
\hline$\varphi_{1}$ & $\begin{array}{c}(0.5,0.7,0.8) \\
(0.1,0.2,0.4) \\
(0.1,0.2,0.3)\end{array}$ & $\begin{array}{c}(0.2,0.4,0.6) \\
(0.3,0.4,0.5) \\
(0.1,0.3,0.4)\end{array}$ & $\begin{array}{c}(0.4,0.5,0.6) \\
(0.2,0.4,0.5) \\
(0.3,0.5,0.7)\end{array}$ & $\begin{array}{c}(0.3,0.5,0.8) \\
(0.4,0.5,0.7) \\
(0.3,0.4,0.5)\end{array}$ \\
\hline$\varphi_{2}$ & $\begin{array}{c}(0.4,0.5,0.7) \\
(0.3,0.6,0.8) \\
(0.4,0.6,0.7)\end{array}$ & $\begin{array}{c}(0.3,0.4,0.5) \\
(0.2,0.4,0.6) \\
(0.3,0.4,0.5)\end{array}$ & $\begin{array}{l}(0.4,0.5,0.7) \\
(0.5,0.6,0.9) \\
(0.3,0.4,0.6)\end{array}$ & $\begin{array}{c}(0.2,0.4,0.6) \\
(0.2,0.3,0.4) \\
(0.1,0.3,0.5)\end{array}$ \\
\hline$\varphi_{3}$ & $\begin{array}{c}(0.4,0.7,0.9) \\
(0.3,0.5,0.8) \\
(0.6,0.8,0.9)\end{array}$ & $\begin{array}{c}(0.1,0.3,0.5) \\
(0.2,0.4,0.7) \\
(0.5,0.8,0.9)\end{array}$ & $\begin{array}{c}(0.2,0.4,0.5) \\
(0.3,0.5,0.7) \\
(0.2,0.4,0.6)\end{array}$ & $\begin{array}{c}(0.4,0.5,0.7) \\
(0.5,0.8,0.9) \\
(0.2,0.3,0.6)\end{array}$ \\
\hline$\varphi_{4}$ & $\begin{array}{c}(0.3,0.4,0.7) \\
(0.3,0.7,0.9) \\
(0.2,0.4,0.5)\end{array}$ & $\begin{array}{c}(0.2,0.8,0.9) \\
(0.4,0.5,0.6) \\
(0.4,0.5,0.7)\end{array}$ & $\begin{array}{c}(0.5,0.7,0.9) \\
(0.3,0.4,0.5) \\
(0.2,0.3,0.4)\end{array}$ & $\begin{array}{c}(0.4,0.5,0.6) \\
(0.2,0.3,0.4) \\
(0.1,0.2,0.3)\end{array}$ \\
\hline$\varphi_{5}$ & $\begin{array}{c}(0.5,0.6,0.7) \\
(0.1,0.4,0.5) \\
(0.2,0.3,0.6)\end{array}$ & $\begin{array}{c}(0.6,0.7,0.9) \\
(0.4,0.5,0.7) \\
(0.3,0.4,0.5)\end{array}$ & $\begin{array}{c}(0.5,0.6,0.8) \\
(0.3,0.4,0.5) \\
(0.4,0.5,0.6)\end{array}$ & $\begin{array}{c}(0.3,0.4,0.7) \\
(0.1,0.4,0.5) \\
(0.1,0.3,0.6)\end{array}$ \\
\hline
\end{tabular}

Table 3. TFNNs evaluation matrix by $d_{3}$.

\begin{tabular}{|c|c|c|c|c|}
\hline & $c_{1}$ & $c_{2}$ & $c_{3}$ & $c_{4}$ \\
\hline$\varphi_{1}$ & $\begin{array}{c}(0.5,0.6,0.8) \\
(0.1,0.2,0.6) \\
(0.1,0.2,0.4)\end{array}$ & $\begin{array}{c}(0.3,0.4,0.6) \\
(0.4,0.5,0.7) \\
(0.2,0.3,0.4)\end{array}$ & $\begin{array}{c}(0.4,0.5,0.8) \\
(0.3,0.4,0.5) \\
(0.3,0.6,0.8)\end{array}$ & $\begin{array}{c}(0.4,0.6,0.8) \\
(0.4,0.6,0.7) \\
(0.3,0.4,0.5)\end{array}$ \\
\hline$\varphi_{2}$ & $\begin{array}{c}(0.4,0.5,0.6) \\
(0.5,0.6,0.7) \\
(0.5,0.6,0.7)\end{array}$ & $\begin{array}{c}(0.3,0.4,0.6) \\
(0.2,0.4,0.5) \\
(0.3,0.4,0.5)\end{array}$ & $\begin{array}{c}(0.3,0.5,0.7) \\
(0.2,0.6,0.9) \\
(0.3,0.4,0.7)\end{array}$ & $\begin{array}{c}(0.3,0.4,0.6) \\
(0.2,0.3,0.5) \\
(0.2,0.3,0.5)\end{array}$ \\
\hline
\end{tabular}


Table 3. Cont.

\begin{tabular}{|c|c|c|c|c|}
\hline & $\mathrm{c}_{1}$ & $c_{2}$ & $c_{3}$ & $\mathrm{c}_{4}$ \\
\hline$\varphi_{3}$ & $\begin{array}{c}(0.5,0.7,0.8) \\
(0.4,0.5,0.7) \\
(0.7,0.8,0.9)\end{array}$ & $\begin{array}{c}(0.2,0.3,0.5) \\
(0.2,0.4,0.5) \\
(0.5,0.7,0.9)\end{array}$ & $\begin{array}{c}(0.3,0.4,0.5) \\
(0.3,0.4,0.6) \\
(0.2,0.4,0.5)\end{array}$ & $\begin{array}{c}(0.3,0.5,0.7) \\
(0.5,0.7,0.9) \\
(0.2,0.3,0.4)\end{array}$ \\
\hline$\varphi_{4}$ & $\begin{array}{c}(0.3,0.4,0.5) \\
(0.3,0.8,0.9) \\
(0.1,0.4,0.5)\end{array}$ & $\begin{array}{c}(0.2,0.5,0.8) \\
(0.4,0.5,0.9) \\
(0.4,0.6,0.7)\end{array}$ & $\begin{array}{c}(0.5,0.6,0.9) \\
(0.3,0.4,0.6) \\
(0.2,0.3,0.5)\end{array}$ & $\begin{array}{c}(0.3,0.5,0.7) \\
(0.2,0.3,0.5) \\
(0.1,0.2,0.4)\end{array}$ \\
\hline$\varphi_{5}$ & $\begin{array}{c}(0.5,0.6,0.8) \\
(0.1,0.4,0.6) \\
(0.2,0.3,0.5)\end{array}$ & $\begin{array}{c}(0.6,0.7,0.8) \\
(0.4,0.5,0.6) \\
(0.3,0.4,0.5)\end{array}$ & $\begin{array}{c}(0.5,0.6,0.7) \\
(0.3,0.4,0.6) \\
(0.4,0.5,0.7)\end{array}$ & $\begin{array}{c}(0.3,0.4,0.5) \\
(0.2,0.4,0.5) \\
(0.1,0.3,0.4)\end{array}$ \\
\hline
\end{tabular}

Step 1. Utilize overall values of $\eta^{\lambda}=\left[\eta_{i j}^{\lambda}\right]_{m \times n}$ to $\eta=\left[\eta_{i j}\right]_{m \times n}$ using the TFNNWA operator; the aggregation results are listed in Table 4 as follows.

Table 4. The aggregation values by TFNNWA operator.

\begin{tabular}{|c|c|c|}
\hline & $c_{1}$ & $c_{2}$ \\
\hline$\varphi_{1}$ & $\begin{array}{c}(0.5376,0.7243,0.8431) \\
(0.1275,0.2305,0.4690) \\
(0.1000,0.2305,0.3514)\end{array}$ & $\begin{array}{c}(0.2566,0.4371,0.6383) \\
(0.3514,0.4522,0.5700) \\
(0.1464,0.3318,0.4325)\end{array}$ \\
\hline$\varphi_{2}$ & $\begin{array}{c}(0.4371,0.5376,0.6822) \\
(0.3675,0.5629,0.7043) \\
(0.3782,0.5206,0.6222)\end{array}$ & $\begin{array}{c}(0.2665,0.4000,0.5577) \\
(0.2305,0.4325,0.6106) \\
(0.2603,0.3617,0.5000)\end{array}$ \\
\hline$\varphi_{3}$ & $\begin{array}{c}(0.3894,0.6413,0.8134) \\
(0.2757,0.4624,0.6608) \\
(0.5805,0.7234,0.8637)\end{array}$ & $\begin{array}{c}(0.1565,0.3000,0.4671) \\
(0.2549,0.4325,0.6201) \\
(0.5329,0.7434,0.8637)\end{array}$ \\
\hline$\varphi_{4}$ & $\begin{array}{c}(0.2665,0.4371,0.6677) \\
(0.3000,0.6812,0.8637) \\
(0.1366,0.3138,0.4181)\end{array}$ & $\begin{array}{c}(0.3213,0.7231,0.8536) \\
(0.3617,0.4624,0.6105) \\
(0.3138,0.5186,0.7000)\end{array}$ \\
\hline \multirow[t]{2}{*}{$\varphi_{5}$} & $\begin{array}{c}(0.5819,0.6862,0.8117) \\
(0.1275,0.3617,0.4796) \\
(0.2000,0.3000,0.5020)\end{array}$ & $\begin{array}{c}(0.5675,0.7000,0.8536), \\
(0.4000,0.5000,0.7112), \\
(0.2603,0.4000,0.5000)\end{array}$ \\
\hline & $c_{3}$ & $\mathrm{c}_{4}$ \\
\hline$\varphi_{1}$ & $\begin{array}{c}(0.4371,0.5376,0.6851) \\
(0.1702,0.3138,0.4181) \\
(0.2603,0.4337,0.5911)\end{array}$ & $\begin{array}{c}(0.3569,0.6001,0.8431) \\
(0.4325,0.5527,0.7335) \\
(0.2603,0.4000,0.5329)\end{array}$ \\
\hline$\varphi_{2}$ & $\begin{array}{l}(0.4195,0.5376,0.7958) \\
(0.4437,0.6333,0.8637) \\
(0.3587,0.4610,0.6531)\end{array}$ & $\begin{array}{c}(0.2957,0.4371,0.6383), \\
(0.2305,0.3318,0.4820), \\
(0.2018,0.3824,0.5894)\end{array}$ \\
\hline$\varphi_{3}$ & $\begin{array}{c}(0.4474,0.5915,0.7153) \\
(0.3000,0.4782,0.6431) \\
(0.2000,0.4000,0.5428)\end{array}$ & $\begin{array}{c}(0.3812,0.5000,0.7397) \\
(0.5000,0.7434,0.9000) \\
(0.2000,0.3000,0.4801)\end{array}$ \\
\hline$\varphi_{4}$ & $\begin{array}{c}(0.4671,0.6486,0.8725) \\
(0.3000,0.4000,0.5186) \\
(0.2000,0.3000,0.4820)\end{array}$ & $\begin{array}{l}(0.4195,0.5819,0.7675), \\
(0.2549,0.3824,0.5331) \\
(0.1275,0.2305,0.3800)\end{array}$ \\
\hline$\varphi_{5}$ & $\begin{array}{c}(0.5000,0.6000,0.7500) \\
(0.2603,0.4000,0.5186) \\
(0.2462,0.4624,0.6188)\end{array}$ & $\begin{array}{c}(0.3000,0.4000,0.7738), \\
(0.1464,0.4000,0.5000) \\
(0.1000,0.3587,0.5533)\end{array}$ \\
\hline
\end{tabular}


Step 2. Compute the values of $\varphi^{+}$(PIS) and $\varphi^{-}$(NIS), for all benefit attributes and based on the Formulas (24) and (25), we can obtain the (PIS) $\varphi^{+}$and (NIS) $\varphi^{-}$as follows.

$$
\begin{gathered}
\varphi^{+}=\left\{\begin{array}{l}
\{(0.5819,0.7243,0.8431),(0.1275,0.2305,0.4690),(0.1000,0.2305,0.3514)\}, \\
\{(0.5675,0.7231,0.8536),(0.2305,0.4325,0.5700),(0.1464,0.3318,0.4325)\}, \\
\{(0.5000,0.6486,0.8725),(0.1702,0.3138,0.4181),(0.2000,0.3000,0.4820)\}, \\
\{(0.4195,0.6001,0.8431),(0.1464,0.3318,0.4820),(0.1000,0.2305,0.3800)\}
\end{array}\right\} \\
\varphi^{-}=\left\{\begin{array}{l}
\{(0.2665,0.4371,0.6677),(0.3675,0.6812,0.8637),(0.5805,0.7234,0.8637)\}, \\
\{(0.1565,0.3000,0.4671),(0.4000,0.5000,0.7112),(0.5329,0.7434,0.8637)\}, \\
\{(0.4195,0.5376,0.6851),(0.4437,0.6333,0.8637),(0.3587,0.4624,0.6531)\}, \\
\{(0.2957,0.4000,0.6383),(0.5000,0.7434,0.9000),(0.2603,0.4000,0.5894)\}
\end{array}\right\}
\end{gathered}
$$

Step 3. Based on Equation (11) and the attribute weighting vector $\omega_{j}$, calculate the values of $\chi_{i}$ and $\psi_{i}$.

$$
\begin{aligned}
& \chi_{1}=0.3101, \chi_{2}=0.6959, \chi_{3}=0.7621, \chi_{4}=0.3877, \chi_{5}=0.3039, \\
& \psi_{1}=0.1738, \psi_{2}=0.2683, \psi_{3}=0.2963, \psi_{4}=0.2486, \psi_{5}=0.1038 .
\end{aligned}
$$

Step 4. Compute the values of $\Omega_{i}$ based on the results of $\chi_{i}$ and $\psi_{i}$; the calculating values are listed as follows. (Let $\alpha=0.6$ )

$$
\Omega_{1}=0.1534, \Omega_{2}=0.8550, \Omega_{3}=1.0000, \Omega_{4}=0.4106, \Omega_{5}=0.0000 .
$$

Step 5. To choose the best alternative by rank the values of $\Omega_{i}$, the ranking of $\Omega_{i}$ is $\Omega_{5}>\Omega_{1}>$ $\Omega_{4}>\Omega_{2}>\Omega_{3}$, and the best alternative is $\varphi_{5}$.

\subsection{Comparative Analyses}

In this section, we compare our proposed extended TFNNs VIKOR model with the TFNNWA and TFNNWG operators defined by Biswas [21].

Based on the values of Table 4 and attributes weighting vector $\omega=(0.42,0.13,0.25,0.30)^{T}$, we can utilize overall $\eta_{i j}$ to $\eta_{i}$ by TFNNWA and TFNNWG operators.

Calculate results $\eta_{i}$ by TFNNWA operator:

$$
\begin{aligned}
& \eta_{1}=\{(0.4720,0.6616,0.8270),(0.1835,0.3051,0.4956),(0.1413,0.2884,0.4196)\} \\
& \eta_{2}=\{(0.4071,0.5302,0.7247),(0.2852,0.4513,0.6269),(0.2672,0.4113,0.5744)\} \\
& \eta_{3}=\{(0.4064,0.5970,0.7779),(0.2930,0.4935,0.6851),(0.3026,0.4654,0.6354)\} \\
& \eta_{4}=\{(0.3941,0.6060,0.8109),(0.2595,0.4589,0.6196),(0.1344,0.2689,0.4126)\} \\
& \eta_{5}=\{(0.5302,0.6414,0.8251),(0.1500,0.3602,0.4843),(0.1508,0.3246,0.5081)\}
\end{aligned}
$$

Calculate results $\eta_{i}$ by TFNNWG operator:

$$
\begin{aligned}
& \eta_{1}=\{(0.3854,0.5761,0.7590),(0.2812,0.4078,0.5965),(0.2060,0.3674,0.5071)\} \\
& \eta_{2}=\{(0.3321,0.4569,0.6516),(0.3634,0.5475,0.7355),(0.2672,0.4113,0.5744)\} \\
& \eta_{3}=\{(0.3238,0.5054,0.6977),(0.3755,0.5954,0.7831),(0.4438,0.6137,0.7741)\} \\
& \eta_{4}=\{(0.3154,0.5166,0.7381),(0.3200,0.5653,0.7461),(0.1873,0.3436,0.4993)\} \\
& \eta_{5}=\{(0.4336,0.5449,0.7733),(0.2185,0.4286,0.5624),(0.2096,0.3963,0.5793)\}
\end{aligned}
$$

Calculating the alternative scores $s\left(\eta_{i}\right)$ by score functions of TFNNs as listed in Table 5 . 
Table 5. Alternative scores $s\left(\eta_{i}\right)$ by TFNNWA and TFNNWG operators.

\begin{tabular}{cc}
\hline TFNNWA Operator & TFNNWG Operator \\
\hline$s\left(\eta_{1}\right)=0.6277, s\left(\eta_{2}\right)=0.5431$, & $s\left(\eta_{1}\right)=0.5692, s\left(\eta_{2}\right)=0.4928$, \\
$s\left(\eta_{3}\right)=0.5299, s\left(\eta_{4}\right)=0.5961$, & $s\left(\eta_{3}\right)=0.4507, s\left(\eta_{4}\right)=0.5444$, \\
$s\left(\eta_{5}\right)=0.6078$. & $s\left(\eta_{5}\right)=0.5546$. \\
\hline
\end{tabular}

The ranking of alternatives by TFNNWA and TFNNWG operators are listed in Table 6 .

Table 6. Rank of alternatives by TFNNWA and TFNNWG operators.

\begin{tabular}{cc}
\hline & Order \\
\hline TFNNWA & $\varphi_{1}>\varphi_{5}>\varphi_{4}>\varphi_{2}>\varphi_{3}$ \\
TFNNWG & $\varphi_{1}>\varphi_{5}>\varphi_{4}>\varphi_{2}>\varphi_{3}$ \\
TFNNs VIKOR & $\varphi_{5}>\varphi_{1}>\varphi_{4}>\varphi_{2}>\varphi_{3}$ \\
\hline
\end{tabular}

Comparing the values of our proposed TFNNs VIKOR method with those of TFNNWA and TFNNWG operators, the results are slightly different in their ranking of the alternatives and the best alternatives are not same. The TFNNs VIKOR method can consider the conflicting attributes and can be more reasonable and scientific in the application of MCGDM problems.

\section{Conclusions}

In our article, we proposed the TFNNs VIKOR method based on the fundamental theories of TFNNs and the original VIKOR model. Firstly, we introduced the concepts, operation formulas and the distance calculating method of TFNNs. Then we reviewed some aggregation operators of TFNNs. Thereafter, the calculating steps of the VIKOR model for TFNNs MCGDM problems were simply presented using our proposed method, which is more scientific and reasonable for considering the conflicting attributes. Furthermore, a numerical example for potential evaluation of emerging technology commercialization has been proposed to illustrate the new method and some comparisons were also conducted to further illustrate the advantages of the new method.

In the future, our proposed TFNN VIKOR model can be applied to risk analysis, MCGDM problems [46-57] and many other uncertain and fuzzy environments [58-74].

Author Contributions: J.W., G.W. and M.L. conceived and worked together to achieve this work, J.W. compiled the computing program by Matlab and analyzed the data, J.W. and G.W. wrote the paper. Finally, all the authors have read and approved the final manuscript.

Funding: The work was supported by the National Natural Science Foundation of China under Grant No. 71571128 and the Humanities and Social Sciences Foundation of Ministry of Education of the People's Republic of China (16XJA630005) and the Construction Plan of Scientific Research Innovation Team for Colleges and Universities in Sichuan Province (15TD0004).

Conflicts of Interest: The authors declare no conflict of interest.

\section{References}

1. Opricovic, S.; Tzeng, G.H. Compromise solution by MCDM methods: A comparative analysis of VIKOR and TOPSIS. Eur. J. Oper. Res. 2007, 156, 445-455. [CrossRef]

2. Wei, G.W. Grey relational analysis method for intuitionistic fuzzy multiple attribute decision making with preference information on alternatives. Int. J. Comput. Intell. Syst. 2011, 4, 164-173. [CrossRef]

3. Wei, G.W. Grey relational analysis model for dynamic hybrid multiple attribute decision making. Knowl. Based Syst. 2011, 24, 672-679. [CrossRef]

4. Wei, G.W. Gray relational analysis method for intuitionistic fuzzy multiple attribute decision making. Expert Syst. Appl. 2011, 38, 11671-11677. [CrossRef]

5. Baležentis, A.; Baležentis, T.; Brauers, W.K.M. Personnel selection based on computing with words and fuzzy MULTIMOORA. Expert Syst. Appl. 2012, 39, 7961-7967. [CrossRef] 
6. Baležentis, T. Group multi-criteria decision making based upon interval-valued fuzzy numbers: An extension of the MULTIMOORA method. Expert Syst. Appl. 2013, 40, 543-550. [CrossRef]

7. Ziemba, P. NEAT F-PROMETHEE-A new fuzzy multiple criteria decision making method based on the adjustment of mapping trapezoidal fuzzy numbers. Expert Syst. Appl. 2018, 110, 363-380. [CrossRef]

8. Hashemi, H.; Mousavi, S.M.; Zavadskas, E.K.; Chalekaee, A.; Turskis, Z. A New Group Decision Model Based on Grey-Intuitionistic Fuzzy-ELECTRE and VIKOR for Contractor Assessment Problem. Sustainability 2018, 10, 1635. [CrossRef]

9. Hwang, C.L.; Yoon, K. Multiple Attribute Decision Making. Economics and Mathematical Systems; Springer: Berlin, Germany, 1981.

10. Chen, C.T.; Lin, C.T.; Huang, S.F. A fuzzy approach for supplier evaluation and selection in supply chain management. Int. J. Prod. Econ. 2006, 102, 89-301. [CrossRef]

11. Zadeh, L.A. Fuzzy sets. Inf. Control 1965, 8, 338-356. [CrossRef]

12. Bellman, R.; Zadeh, L.A. Decision making in a fuzzy environment. Manag. Sci. 1970, 17, 141-164. [CrossRef]

13. Yager, R.R. Multiple objective decision-making using fuzzy sets. Int. J. Man-Mach. Stud. 1997, 9, $375-382$. [CrossRef]

14. Smarandache, F. A Unifying Field in Logics. Neutrosophy: Neutrosophic Probability, Set and Logic; American Research Press: Rehoboth, NM, USA, 1999.

15. Smarandache, F. A Unifying Field in Logics: Neutrosophic Logic. Neutrosophy, Neutrosophic Set, Neutrosophic Probability and Statistics, 3rd ed.; American Research Press: Phoenix, AZ, USA, 2003.

16. Wang, H.; Smarandache, F.; Zhang, Y.Q.; Sunderraman, R. Single valued neutrosophic sets. Multispace Multistruct 2010, 4, 410-413.

17. Wang, H.; Smarandache, F.; Zhang, Y.Q.; Sunderraman, R. Interval Neutrosophic Sets and Logic: Theory and Applications in Computing; Hexis: Phoenix, AZ, USA, 2005.

18. Wang, J.; Tang, X.; Wei, G. Models for Multiple Attribute Decision-Making with Dual Generalized Single-Valued Neutrosophic Bonferroni Mean Operators. Algorithms 2018, 11, 2. [CrossRef]

19. Wang, J.; Wei, G.; Wei, Y. Models for Green Supplier Selection with Some 2-Tuple Linguistic Neutrosophic Number Bonferroni Mean Operators. Symmetry 2018, 10, 131. [CrossRef]

20. Wu, S.; Wang, J.; Wei, G.; Wei, Y. Research on Construction Engineering Project Risk Assessment with Some 2-Tuple Linguistic Neutrosophic Hamy Mean Operators. Sustainability 2018, 10, 1536. [CrossRef]

21. Biswas, P.; Pramanik, S.; Gir, B.C. Aggregation of triangular fuzzy neutrosophic set information and its application to multi-attribute decision making. Neutrosophic Sets Syst. 2016, 12, 22-40.

22. Şahin, M.; Ecemiş, O.; Uluçay, V.; Kargın, A. Some new generalized aggregation operators based on centroid single valued triangular neutrosophic numbers and their applications in multiattribute decision making. Asian J. Math. Comput. Res. 2017, 16, 63-84.

23. Aal, S.I.; Ellatif, M.M.; Hassan, M.M. Proposed Model for Evaluating Information Systems Quality Based on Single Valued Triangular Neutrosophic Numbers. Int. J. Math. Sci. Comput. 2018, 4, 1-14.

24. Ye, J. Trapezoidal neutrosophic set and its application to multiple attribute decision-making. Neural Comput. Appl. 2015, 26, 1157-1166. [CrossRef]

25. Biswas, P.; Pramanik, S.; Giri, B.C. Value and ambiguity index based ranking method of single-valued trapezoidal neutrosophic numbers and its application to multi-attribute decision making. Neutrosophic Sets Syst. 2016, 12, 127-138.

26. Tan, R.; Zhang, W. Multiple attribute group decision making methods based on trapezoidal fuzzy neutrosophic numbers. J. Intell. Fuzzy Syst. 2017, 33, 1-18. [CrossRef]

27. Opricovic, S.; Tzeng, G.-H. Extended VIKOR method in comparison with outranking methods. Eur. J. Oper. Res. 2007, 178, 514-529. [CrossRef]

28. Cevikcan, E.; Çebi, S.; Kaya, I. Fuzzy VIKOR and Fuzzy Axiomatic Design Versus to Fuzzy Topsis: An Application of Candidate Assessment. Mul. Valued Log. Soft Comput. 2009, 15, 181-208.

29. Bausys, R.; Zavadskas, E.K. Multicriteria decision making approach by VIKOR under interval neutrosophic set environment. Econ. Comput. Econ. Cybern. Stud. Res. 2015, 4, 33-48.

30. Park, J.H.; Cho, H.J.; Kwun, Y.C. Extension of the VIKOR method for group decision making with interval-valued intuitionistic fuzzy information. Fuzzy Optim. Decis. Mak. 2011, 10, 233-253. [CrossRef]

31. Selvakumari, K.; Priyadharshini, M.A. VIKOR method for decision making problem using octagonal neutrosophic soft matrix. Int. J. Latest Eng. Res. Appl. 2017, 2, 41-45. 
32. Wan, S.; Wang, Q.; Dong, J. The extended VIKOR method for multi-attribute group decision making with triangular intuitionistic fuzzy numbers. Knowl. Based Syst. 2013, 52, 65-77. [CrossRef]

33. Liu, H.; You, J.; Fan, X.; Chen, Y. Site selection in waste management by the VIKOR method using linguistic assessment. Appl. Soft Comput. 2014, 21, 453-461. [CrossRef]

34. Qin, J.; Liu, X.; Pedrycz, W. An extended VIKOR method based on prospect theory for multiple attribute decision making under interval type-2 fuzzy environment. Knowl. Based Syst. 2015, 86, 116-130. [CrossRef]

35. Chen, T.Y. Remoteness index-based Pythagorean fuzzy VIKOR methods with a generalized distance measure for multiple criteria decision analysis. Inf. Fusion 2018, 41, 129-150. [CrossRef]

36. Liao, H.; Xu, Z.; Zeng, X. Hesitant Fuzzy Linguistic VIKOR Method and Its Application in Qualitative Multiple Criteria Decision Making. IEEE Trans. Fuzzy Syst. 2015, 23, 1343-1355. [CrossRef]

37. Ren, Z.; Xu, Z.; Wang, H. Dual hesitant fuzzy VIKOR method for multi-criteria group decision making based on fuzzy measure and new comparison method. Inf. Sci. 2017, 388, 1-16. [CrossRef]

38. Li, Z.; Liu, P.; Qin, X. An extended VIKOR method for decision making problem with linguistic intuitionistic fuzzy numbers based on some new operational laws and entropy. J. Intell. Fuzzy Syst. 2017, 33, 1919-1931. [CrossRef]

39. Pouresmaeil, H.; Shivanian, E.; Khorram, E.; Fathabadi, H.S. An extended method using TOPSIS and VIKOR for multiple attribute decision making with multiple decision makers and single valued neutrosophic numbers. Adv. Appl. Stat. 2017, 50, 261-292. [CrossRef]

40. Huang, Y.H.; Wei, G.W.; Wei, C. VIKOR method for interval neutrosophic multiple attribute group decision-making. Information 2018, 8, 144. [CrossRef]

41. Zhang, N.; Wei, G. Extension of VIKOR method for decision making problem based on hesitant fuzzy set. Appl. Math. Model. 2013, 37, 4938-4947. [CrossRef]

42. Opricovic, S. Multicriteria Optimization of Civil Engineering Systems. Ph.D. Thesis, Faculty of Civil Engineering, Belgrade, Serbia, 1998.

43. Wei, G.; Zhang, N. A multiple criteria hesitant fuzzy decision making with Shapley value-based VIKOR method. J. Intell. Fuzzy Syst. 2014, 26, 1065-1075.

44. Wei, G.; Zhang, N. Extension of VIKOR method for decision making problem based on hesitant fuzzy set. J. Intell. Fuzzy Syst. 2013, 37, 1065-1075.

45. Deng, X.M.; Wei, G.; Gao, H.; Wang, J. Models for safety assessment of construction project with some 2-tuple linguistic Pythagorean fuzzy Bonferroni mean operators. IEEE Access 2018, 6, 52105-52137. [CrossRef]

46. Merigo, J.M.; Casanovas, M.; Martínez, L. Linguistic aggregation operators for linguistic decision making based on the Dempster-Shafer theory of Evidence. Int. J. Uncertain. Fuzz. Knowl. Based Syst. 2010, 18, $287-304$. [CrossRef]

47. Wei, G.; Gao, H.; Wei, Y. Some q-Rung Orthopair Fuzzy Heronian Mean Operators in Multiple Attribute Decision Making. Int. J. Intell. Syst. 2018, 33, 1426-1458. [CrossRef]

48. Wei, G.; Lu, M.; Tang, X.; Wei, Y. Pythagorean Hesitant Fuzzy Hamacher Aggregation Operators and Their Application to Multiple Attribute Decision Making. Int. J. Intell. Syst. 2018, 33, 1197-1233. [CrossRef]

49. Merigo, J.M.; Gil-Lafuente, A.M. Fuzzy induced generalized aggregation operators and its application in multi-person decision making. Expert Syst. Appl. 2011, 38, 9761-9772. [CrossRef]

50. Wei, G.W. Some similarity measures for picture fuzzy sets and their applications. Iran. J. Fuzzy Syst. 2018, 15, 77-89.

51. Wu, S.; Wang, J.; Wei, G.; Wei, Y. Research on Construction Engineering Project Risk Assessment with Some 2-Tuple Linguistic Neutrosophic Hamy Mean Operators. Sustainability 2018, 10, 1536. [CrossRef]

52. Wang, J.; Wei, G.; Lu, M. TODIM Method for Multiple Attribute Group Decision Making under 2-Tuple Linguistic Neutrosophic Environment. Symmetry 2018, 10, 486. [CrossRef]

53. Chen, T.Y.; Li, C.H. Determining objective weights with intuitionistic fuzzy entropy measures: A comparative analysis. Inf. Sci. 2010, 180, 4207-4222. [CrossRef]

54. Kuo, M.S.; Liang, G.S. A soft computing method of performance evaluation with MCDM based on interval-valued fuzzy numbers. Appl. Soft Comput. 2012, 12, 476-485. [CrossRef]

55. Bolukbas, U.; Guneri, A.F. Knowledge-based decision making for the technology competency analysis of manufacturing enterprises. Appl. Soft Comput. 2018, 67, 781-799. [CrossRef]

56. Tang, X.Y.; Wei, G.W. Models for green supplier selection in green supply chain management with Pythagorean 2-tuple linguistic information. IEEE Access 2018, 6, 18042-18060. [CrossRef] 
57. Kamis, N.H.; Chiclana, F.; Levesley, J. Preference similarity network structural equivalence clustering based consensus group decision making model. Appl. Soft Comput. 2018, 67, 706-720. [CrossRef]

58. Wei, G.W.; Lu, M. Pythagorean Fuzzy Maclaurin Symmetric Mean Operators in multiple attribute decision making. Int. J. Intell. Syst. 2018, 33, 1043-1070. [CrossRef]

59. Gao, H.; Wei, G.W.; Huang, Y.H. Dual hesitant bipolar fuzzy Hamacher prioritized aggregation operators in multiple attribute decision making. IEEE Access 2018, 6, 11508-11522. [CrossRef]

60. Ullah, A.M.M.S.; Noor-E-Alam, M. Big data driven graphical information based fuzzy multi criteria decision making. Appl. Soft Comput. 2018, 63, 23-38. [CrossRef]

61. Gao, H. Pythagorean Fuzzy Hamacher Prioritized Aggregation Operators in Multiple Attribute Decision Making. J. Intell. Fuzzy Syst. 2018, 35, 2229-2245. [CrossRef]

62. Wei, G.; Wei, Y. Some single-valued neutrosophic dombi prioritized weighted aggregation operators in multiple attribute decision making. J. Intell. Fuzzy Syst. 2018, 35, 2001-2013. [CrossRef]

63. Huang, Y.H.; Wei, G.W. TODIM Method for Pythagorean 2-tuple Linguistic Multiple Attribute Decision Making. J. Intell. Fuzzy Syst. 2018, 35, 901-915. [CrossRef]

64. Krishnamurthy, M.; Marcinek, P.; Malik, K.M.; Afzal, M. Representing Social Network Patient Data as Evidence-Based Knowledge to Support Decision Making in Disease Progression for Comorbidities. IEEE Access 2018, 6, 12951-12965. [CrossRef]

65. Wei, G.W.; Gao, H. The generalized Dice similarity measures for picture fuzzy sets and their applications. Informatica 2018, 29, 107-124. [CrossRef]

66. Gao, H.; Lu, M.; Wei, G.W.; Wei, Y. Some novel Pythagorean fuzzy interaction aggregation operators in multiple attribute decision making. Fundam. Inform. 2018, 159, 385-428. [CrossRef]

67. Garg, H.; Arora, R. Generalized and group-based generalized intuitionistic fuzzy soft sets with applications in decision-making. Appl. Intell. 2018, 48, 343-356. [CrossRef]

68. Wei, G.W.; Alsaadi, F.E.; Hayat, T.; Alsaedi, A. Bipolar fuzzy Hamacher aggregation operators in multiple attribute decision making. Int. J. Fuzzy Syst. 2018, 20, 1-12. [CrossRef]

69. Wei, G.W.; Gao, H.; Wang, J.; Huang, Y.H. Research on Risk Evaluation of Enterprise Human Capital Investment with Interval-valued bipolar 2-tuple linguistic Information. IEEE Access 2018, 6, 35697-35712. [CrossRef]

70. Rahman, M.A.; Mezhuyev, V.; Bhuiyan, M.Z.; Sadat, S.N.; Zakaria, S.A.; Refat, N. Reliable Decision Making of Accepting Friend Request on Online Social Networks. IEEE Access 2018, 6, 9484-9491. [CrossRef]

71. Wei, G.W.; Wei, Y. Similarity measures of Pythagorean fuzzy sets based on cosine function and their applications. Int. J. Intell. Syst. 2018, 33, 634-652. [CrossRef]

72. Lee, H.; Kim, S. Black-Box Classifier Interpretation Using Decision Tree and Fuzzy Logic-Based Classifier Implementation. Int. J. Fuzzy Logic Intell. Syst. 2016, 16, 27-35. [CrossRef]

73. Grzegorzewski, P. On Separability of Fuzzy Relations. Int. J. Fuzzy Logic Intell. Syst. 2017, 17, 137-144. [CrossRef]

74. Ziemba, P.; Jankowski, J.; Watróbski, J. Online Comparison System with Certain and Uncertain Criteria Based on Multi-criteria Decision Analysis Method. In Computational Collective Intelligence; Nguyen, N., Papadopoulos, G., Jędrzejowicz, P., Trawiński, B., Vossen, G., Eds.; Springer: Cham, Switzerland, 2017; Volume 10449, pp. 579-589.

(C) 2018 by the authors. Licensee MDPI, Basel, Switzerland. This article is an open access article distributed under the terms and conditions of the Creative Commons Attribution (CC BY) license (http://creativecommons.org/licenses/by/4.0/). 